\title{
EPRBioDose 2013: EPR applications and biological dosimetry
}

\author{
A. Wieser · F. Darroudi
}

Received: 24 February 2014 / Accepted: 27 February 2014/Published online: 19 March 2014

(C) Springer-Verlag Berlin Heidelberg 2014

The series of EPRBioDose conferences includes both the International Symposium on EPR Dosimetry and Dating (EPR) and the International Conference on Biological Dosimetry (BioDose). EPRBioDose 2013 was jointly organized by Leiden University Medical Center (Leiden, The Netherlands) and the Helmholtz Zentrum München (München, Germany) and was held on 24-28 March 2013 in Leiden. The conference topics covered new aspects of traditional EPR dosimetry applications, retrospective dosimetry with physical and cytogenetic techniques, dosimetry for mass casualty events and technical developments in particularly on automatic analysing systems for biological assays, as well as those applicable for dose assessment in scenarios of mass casualties. The aim of the conference was to share technological advances achieved since the last EPRBioDose conference and to improve the understanding of physical, biophysical and biological processes that can be used in the dosimetry of ionizing radiation. This volume of Radiation and Environmental Biophysics includes fifteen papers that were presented at the conference.

\section{Traditional EPR dosimetry applications}

In the past, EPR conferences were held in an effort to provide a platform for presenting new developments in EPR

\footnotetext{
A. Wieser $(\bowtie)$

Helmholtz Zentrum München - German Research Center for Environmental Health, Institute of Radiation Protection, 85764 Neuherberg, Germany

e-mail: wieser@helmholtz-muenchen.de

F. Darroudi

Department of Toxicogenetics, Leiden University Medical

Centre (LUMC), Einthovenweg 20, 2300RC Leiden,

The Netherlands

e-mail: f.darroudi@lumc.nl
}

spectrometry, for measurement of absorbed doses in objects of geological, paleontological and archaeological interests with the aim to assess their age. Nowadays, EPR is well established in dating, and it is an important tool for solving dating problems beyond an age of about 60,000 years, the age limit for radio-carbon dating (Skinner 2011). At this conference, EPR applications were presented on paleontological dating of fossil mammals teeth, crustaceans, molluscs and corals, geological dating with quartz and submarine depositions, and material characterization of obsidians and archaeological ceramics. A number of these presentations will be published in a special issue of radiation protection dosimetry.

In earlier years, a further main topic discussed at EPR symposia was the investigation of new detector materials and the comprehensive analysis of known materials for use in EPR dosimetry for industrial and clinical applications. One of the most intensively investigated detector materials for EPR dosimetry is the amino acid alanine. At this conference, applications of the alanine/EPR dosimetry system were presented as a reference in radiation metrology, for quality assurance in clinical applications and for dosimetry at treatments in teletherapy, with ion beams and neutrons. The challenges and opportunities for extending alanine/ EPR dosimetry into clinical applications were reviewed by Baffa and Kinoshita (2014).

\section{Retrospective dosimetry with physical and cytogenetic techniques}

Reconstruction of individual doses is typically based on the assessment of stable radiation end products in human tissues or liquids following exposure to ionizing radiation. The most important tissue for physical dose reconstruction 
is tooth enamel in which stable radicals are produced and evaluated by EPR spectrometry. In contrast, in biological dose reconstruction, chromosomal damage in peripheral blood lymphocytes is evaluated by means of cytogenetic techniques and optical microscopy. Due to the limited halflife of lymphocytes in peripheral blood, however, assays for assessing chromosomal damages as dicentrics (DC), premature chromosome condensation fragments (PCC) and micronucleus frequency ( $\mathrm{MN})$ are applicable for more recent exposures only. In addition, they allow detection of partial-body exposure with the PCC assay and - for doses $<100$ mGy - with the MN assay (Ainsbury et al. 2011). At EPRBioDose 2013, recent applications of these and other assays for assessment of occupational doses and doses to the population were presented.

For example, a high radiation yield, shortly after exposure, is known for protein biomarkers which are signalling DNA damage. A promising marker for exposure to ionizing radiation is the phosphorylated histone $\gamma-\mathrm{H} 2 \mathrm{AX}$ formed at the site of DNA double-strand breaks (DSB). Foci of $\gamma-\mathrm{H} 2 \mathrm{AX}$ can be detected by immunofluorescence microscopy and, accordingly, DSB repair in radiotherapy patients was quantified by $\gamma$-H2AX detection for the investigation of their clinical radiosensitivity (Chua et al. 2014).

For the reconstruction of doses due to exposures which occurred many years ago, a new cytogenetic assay was proposed based on the detection of chromosomal inversions in peripheral blood lymphocytes with the use of directional genomic hybridization (Ray et al. 2014). The yield of these inversions was found to depend dramatically on the linear energy transfer (LET) of the radiation. This finding is important because it may indicate the potential to discriminate between exposures with different radiation qualities. The current assay of choice for the evaluation of exposures that happened years or decades ago is the detection of stable chromosomal translocations in peripheral blood lymphocytes with single-colour fluorescence in situ hybridization (sFISH). Intrachromosomal and complex aberrations as biomarkers of high LET exposure, as from plutonium exposure of nuclear workers, may be detected with multicolour FISH (mFISH). In a study with workers of the Mayak Production Association with ${ }^{239} \mathrm{Pu}$ burden, mFISH analysis revealed a considerable number of cells with complex chromosomal rearrangements (Sotnik et al. 2014). The issue of estimating radiation doses with FISH in cases of incorporation of radionuclides or combined external and internal exposures has been reviewed by Ainsbury et al. (2014).

Stable radiation-induced radicals are very efficiently produced by ionizing radiation in tooth enamel. Therefore, EPR spectrometry with tooth enamel is particularly suitable for the reconstruction of exposures to the population and radiation workers that dated back several years or decades. The methods of choice for the reconstruction of historic or chronically exposures are EPR spectrometry with tooth enamel and the FISH assay with peripheral blood lymphocytes. Presented were applications to reconstruct doses of professionals chronically exposed more recently, and doses of populations exposed historically because they lived close to the nuclear test site at Semipalatinsk, Kazakhstan, and the plutonium production site Mayak, Russia. An EPR analysis of the absorbed dose in teeth from citizens of Ozyorsk, the residence of the Mayak workers and their families, was presented by Wieser et al. (2014). The populations in the Urals and the Mayak workers represent important epidemiological cohorts and dose reconstruction by means of EPR and FISH are both applied to evaluate and validate the dosimetry systems developed for these cohorts. The current results of FISH analysis of translocations induced by chronic exposure due to ${ }^{90} \mathrm{Sr}$ for the validation of the Techa River Dosimetry System were presented by Vozilova et al. (2014). The recently improved Mayak Worker Dosimetry System 2008 was described by Moseeva et al. (2014), and the risk of circulatory diseases among Mayak workers on basis of this dosimetry system was presented.

\section{Retrospective dosimetry for mass casualty events}

After catastrophic radiological events including radiation injuries of a large number of individuals, their individual absorbed dose needs to be quickly surveyed, in order to decide on the required individual medical treatment and to manage the catastrophe. Biological and physical dose reconstruction methods are currently under evaluation in terms of their ability to comply with this ambitious task. The challenge is-for any biodosimetry technique-to find the best compromise between throughput of samples and accuracy of estimated doses. An overview of the principles and the requirements to biodosimetry for mass casualty events was presented by Swartz et al. (2014).

Cytogenetic and genetic techniques are currently most intensively investigated for use in retrospective dosimetry for large-scale radiological events. Changes in gene expression were found to be highly sensitive to radiation exposure, but their short half-life is disadvantageous. Gene expression might show some potential for use in biodosimetry, but requires more investigations to understand power and limitations of this technique for dosimetry (Paul and Amundson 2008). Traditional cytogenetic assays are currently the most accepted methods for biodosimetry, although they are time consuming and hence inconvenient for dosimetric use in mass casualty events. However, dose evaluation can be accelerated by the automation of the methods for cytogenetic analysis as was demonstrated by Rodrigues et al. (2014). In order to evaluate possibilities to 
increase laboratory capacities in DC scoring, experiences within a network for web-based scoring of images from metaphase spreads were reported by Romm et al. (2014).

A rapid method for triage of highly exposed victims during large-scale radiation emergencies is the detection of protein biomarkers using flow cytometry. The ratio of $\gamma-\mathrm{H} 2 \mathrm{AX}$ foci in lymphocytes to those in granulocytes was presented as promising biomarker for quick dose assessment provided that blood samples were collected within $24 \mathrm{~h}$ after exposure (Wang et al. 2014). An automated biodosimetry tool with high throughput was developed for use following large-scale radiological events on the basis of measurements of fingerstick blood samples. Attempts were presented to extend this tool to measure the kinetics of DNA repair proteins like $\gamma$-H2AX (Turner et al. 2014).

Measurements for dose reconstruction with physical methods are fast and allow for a high throughput of samples. However, dose reconstruction with physical methods for mass casualty events is complicated due to availability of suitable detector materials. EPR spectrometry with tooth enamel has very high radiation sensitivity, but teeth need to be extracted before measurement, and will probably not be available for measurements in mass casualty events. In order to overcome this difficulty, attempts were presented to measure small tooth enamel biopsy samples with Q-band EPR spectrometry (Romanyukha et al. 2014). Another option is in vivo EPR tooth dosimetry that is considered having potential for triage in large-scale accidents. Presented were the current state of EPR equipment development and state-of-the-art performance evaluated on the basis of tests in the laboratory and of practical applications to unexposed persons and radiotherapy patients (Williams et al. 2014). Alternatively to teeth, nails are also being investigated for use in EPR dosimetry. Nails are less radiation sensitive than teeth, but nevertheless have some potential for use in dosimetry. The state of the art in nail dosimetry was presented by Trompier et al. (2014). Alternatively to the tissues of the human body, materials present in ubiquitous personal items and their dosimetric properties are currently being widely investigated. One of these materials is glass found in the displays of electronic portable devices such as mobile phones, which probably represent the most ubiquitous personal items that can be used as a dosimeter, in a large part of the world. The power and current limitations of EPR measurements of glass from touch screens in triage applications were presented by Fattibene et al. (2014).

\section{References}

Ainsbury EA, Bakhanova E, Barquinero JF, Brai M, Chumak V, Correcher V, Darroudi F, Fattibene P, Gruel G, Guclu I, Horn S,
Jaworska A, Kulka U, Lindholm C, Lloyed D, Longo A, Marrale M, Gil OM, Oestereicher U, Pajic J, Rakic B, Romm H, Trompier F, Veronese I, Voisin P, Vral A, Whitehouse CA, Wieser A, Woda C, Wojcik A, Rothkamm K (2011) Review of retrospective dosimetry techniques for external ionising radiation exposures. Radiat Prot Dosim 147:573-592

Ainsbury EA, Moquet J, Rothkamm K, Darroudi F, Vozilova A, Degteva M, Azizova TV, Lloyd DC, Harrison J (2014) What radiation dose does the FISH translocation assay measure in cases of incorporated radionuclides for the Southern Urals populations? Radiat Prot Dosim (submitted)

Baffa O, Kinoshita A (2014) Clinical applications of alanine/electron spin resonance dosimetry. Radiat Environ Biophys. doi:10.1007/ s00411-013-0509-2

Chua MLK, Horn S, Somaiah N, Davies S, Gothard L, A'Hern R, Yarnold J, Rothkamm K (2014) DNA double-strand break repair and induction of apoptosis in ex vivo irradiated blood lymphocytes in relation to late normal tissue effects following breast radiotherapy. Radiat Environ Biophys. doi:10.1007/s00411-0140531-Z

Fattibene P, Trompier F, Wieser A, Brai M, Ciesielski B, De Angelis C, Della Monaca S, Garcia T, Gustafsson H, Hole EO, Juniewicz M, Krefft K, Longo A, Leveque P, Lund E, Marrale M, Michalec B, Mierzwińska G, Rao JL, Romanyukha AA, Tuner H (2014) EPR dosimetry intercomparison using smartphone touch screen glass. Radiat Environ Biophys. doi:10.1007/s00411-014-0533-x

Moseeva MB, Azizova TV, Grigoryeva ES, Haylock R (2014) Risk of circulatory diseases among Mayak PA workers with radiation doses estimated using the improved Mayak Worker Dosimetry System 2008. Radiat Environ Biophys. doi:10.1007/s00411-0140517-x

Paul S, Amundson SA (2008) Development of gene expression signatures for practical radiation biodosimetry. Int $\mathrm{J}$ Radiat Oncol Bio Phys 71:1236-1244

Ray FA, Robinson E, McKenna M, Hada M, George K, Cucinotta F, Goodwin EH, Bedford JS, Bailey SM, Cornforth MN (2014) Directional genomic hybridization: inversions as a potential biodosimeter for retrospective radiation exposure. Radiat Environ Biophys. doi:10.1007/s00411-014-0513-1

Rodrigues M, Beaton LA, Kutzner BC, Wilkins RC (2014) Automated analysis of the cytokinesis-block micronucleus assay for radiation biodosimetry using imaging flow cytometry. Radiat Environ Biophys. doi:10.1007/s00411-014-0525-x

Romanyukha A, Tromier F, Reyes RA (2014) Q-band electron paramagnetic resonance dosimetry in tooth enamel: biopsy procedure and determination of dose detection limit. Radiat Environ Biophys. doi:10.1007/s00411-013-0511-8

Romm H, Ainsbury E, Bajinskis A, Barnard S, Barquinero JF, Barrios L, Beinke C, Puig-Casanovas R, Deperas-Kaminska M, Gregoire E, Kulka U, Oestereicher U, Lindholm C, Moquet J, Rothkamm K, Sommer S, Thierens H, Vral A, Vandersickel V, Wojcik A (2014) Web-based scoring of the dicentric assay, a collaborative biodosimetric scoring strategy for population triage in large scale radiation accidents. Radiat Environ Biophys. doi:10.1007/ s00411-014-0519-8

Skinner AR (2011) Current topics in ESR dating. Radiat Meas 46:749-753

Sotnik NV, Osovets SV, Scherthan H, Azizova TV (2014) mFISH analysis of Chromosome Aberrations in Workers Occupationally Exposed to Mixed Radiation. Radiat Environ Biophys. doi:10. 1007/s00411-014-0536-7

Swartz HM, Williams BB, Flood AB (2014) Overview of the principles and practice of biodosimetry. Radiat Environ Biophys. doi:10.1007/s00411-014-0522-0

Trompier F, Romanyukha A, Reyes R, Vezin H, Queinnec F, Gourier $\mathrm{D}$ (2014) State of the art in nail dosimetry: free radicals 
identification and reaction mechanisms. Radiat Environ Biophys. doi:10.1007/s00411-014-0512-2

Turner HC, Sharma P, Perrier JR, Bertucci A, Smilenov L, Johnson G, Taveras M, Brenner DJ, Garty G (2014) The RABiT: highthroughput technology for assessing global BSB repair. Radiat Environ Biophys. doi:10.1007/s00411-014-0514-0

Vozilova AV, Shagina NB, Degteva MO, Moquet J, Ainsbury EA, Darroudi F (2014) FISH analysis of translocations induced by chronic exposure to Sr radioisotopes: Second set of analysis of the Techa River Cohort. Radiat Prot Dosim (submitted)

Wang Z, Hu H, Hu M, Zhang X, Wang Q, Qiao Y, Liu H, Shen L, Zhou $P$, Chen Y (2014) Ratio of $\gamma$-H2AX level in Lymphocytes to that in granulocytes detected using flow cytometry as a potential biodosimeter for radiation exposure. Radiat Environ Biophys. doi:10.1007/s00411-014-0530-0

Wieser A, Vasilenko E, Aladova E, Fattibene P, Semiochkina N, Smetanin M (2014) Electron paramagnetic resonance measurements of absorbed dose in teeth from citizens of Ozyorsk. Radiat Environ Biophys. doi:10.1007/s00411-014-0527-8

Williams BB, Flood AB, Salikhov I, Kobayashi1 K, Dong R, Rychert K, Du G, Schreiber W, Swartz HM (2014) In vivo EPR tooth dosimetry for triage after a radiation event involving large populations. Radiat Environ Biophys. doi:10.1007/s00411-0140534-9 